\title{
Trimethylsilyl tag for probing protein-ligand interactions by NMR
}

Walter Becker, ${ }^{1,2}$ Luke A. Adams, ${ }^{3}$ Bim Graham, ${ }^{3}$ Gabriel E. Wagner, ${ }^{4}$ Klaus Zangger, ${ }^{2}$ Gottfried Otting, ${ }^{1, *}$ and Christoph Nitsche ${ }^{1, *}$

${ }^{1}$ Research School of Chemistry, Australian National University, Canberra, ACT 2601, Australia ${ }^{2}$ Institute of Chemistry, University of Graz, 8010 Graz, Austria

${ }^{3}$ Monash Institute of Pharmaceutical Sciences, Monash University, Parkville, VIC 3052, Australia

${ }^{4}$ Institute of Hygiene, Microbiology and Environmental Medicine, Medical University of Graz, 8010 Graz, Austria

*gottfried.otting@anu.edu.au

* christoph.nitsche@anu.edu.au

\begin{abstract}
Protein-ligand titrations can readily be monitored with a trimethylsilyl (TMS) tag. Owing to the intensity, narrow line shape and unique chemical shift of a TMS group, dissociation constants can be determined from straightforward 1D ${ }^{1} \mathrm{H}-\mathrm{NMR}$ spectra not only in the fast but also in the slow exchange limit. The tag is easily attached to cysteine residues and a sensitive reporter of ligand binding also at sites where it does not interfere with ligand binding or catalytic efficiency of the target protein. Its utility is demonstrated for the Zika virus NS2B-NS3 protease and the human prolyl isomerase FK506 binding protein.
\end{abstract}

\section{Keywords}

Dissociation constant; lead discovery; protein-ligand interactions; slow exchange; trimethylsilyl group 


\section{Introduction}

Drug discovery critically depends on the identification of suitable lead compounds among the vast pool of candidates evaluated during extensive screening or elaboration campaigns. Specificity of binding and binding affinity to the drug target are two essential parameters defining a lead compound. Nuclear magnetic resonance (NMR) spectroscopy is uniquely positioned to assess both parameters simultaneously and, consequently, has become an important tool in the pharmaceutical industries to screen, identify and validate small molecules as potential lead candidates (Gossert and Jahnke 2016). In recent years, NMR spectroscopy has gained particular importance in the early stages of the drug discovery process, where fragmentbased lead discovery has proven an efficient approach to identify small ligands that bind to the target biomolecule (Doak et al. 2016). In this context, the main focus is often on weakly binding ligand molecules that are in rapid exchange between bound and free state. In subsequent stages

of the drug discovery process, however, alternative methods are necessary to analyze the binding epitope and determine the affinity of elaborated ligands, which are usually in slow chemical exchange on the NMR time scale (Nitsche and Otting 2018).

Although 2D NMR spectra with uniformly ${ }^{15} \mathrm{~N}$-labeled target proteins are considered to be the NMR 'gold standard' to assess ligand binding in both early and later stages of the drug discovery process, several alternative strategies have been developed that do not rely on expensive isotope labeling and require significantly smaller protein quantities. A prominent example is the use of $1 \mathrm{D}{ }^{19} \mathrm{~F}-\mathrm{NMR}$ spectroscopy to detect fluorine-substituted tryptophan, trifluoromethoxy-L-phenylalanine, or cysteine residues, that were post-translationally furnished with a $\mathrm{CF}_{3}$ group, in the target protein (Jackson et al. 2007; Cellitti et al. 2008; Arntson and Pomerantz 2016; Liu et al. 2012). The large chemical shift anisotropy associated with ${ }^{19} \mathrm{~F}$ spins imparts high sensitivity towards changes in the environment but is also a source of line broadening. ${ }^{1} \mathrm{H}-\mathrm{NMR}$ spectra offer intrinsically higher sensitivity and, in practice, most NMR spectrometers are equipped for ${ }^{1} \mathrm{H}-\mathrm{NMR}$ but not always for ${ }^{19} \mathrm{~F}-\mathrm{NMR}$ spectroscopy. The greater sensitivity of ${ }^{1} \mathrm{H}-\mathrm{NMR}$ spectra, however, comes at the expense of spectral overlap between protein and ligand signals, which makes it harder to quantify the protein-ligand interactions from 1D NMR spectra. To a large extent, this problem can be alleviated by labels with a tertiary butyl 
$(t \mathrm{Bu})$ group that produces an exceptionally narrow and intense NMR signal arising from nine methyl protons with degenerate chemical shift. This often makes it possible to assign and track $t \mathrm{Bu}$ signals in the presence of modest spectral overlap with protein signals. Recent examples have demonstrated this for ligands containing a $t \mathrm{Bu}$ group and proteins containing a siteselectively incorporated $t$ Bu-tyrosine residue (Chen et al. 2015; Chen et al. 2016; Jabar et al. 2017). When the spectral overlap is pronounced, however, the ${ }^{1} \mathrm{H}-\mathrm{NMR}$ signal even of a $t \mathrm{Bu}$ group may be difficult to identify and track. Even more limiting, spectral overlap prevents accurate integration of the $t \mathrm{Bu}$ resonance, compromising quantitative evaluation of any titration experiments, where the ligand is in slow exchange between bound and free state.

Here we present a strategy that relies on the unique ${ }^{1} \mathrm{H}$ chemical shift of a trimethylsilyl (TMS) group near 0 ppm, where spectral overlap with protein signals is much less severe than at the chemical shift of a $t \mathrm{Bu}$ group (which is at about $1.3 \mathrm{ppm}$ ). Previously, we investigated the possibility of site-specific incorporation of $p$-TMS-phenylalanine (TMSf) by genetic encoding but, due to ring currents, the TMS signal appeared at about 0.3 rather than $0 \mathrm{ppm}$ and the amino acid could not be incorporated in high yield (Loh et al. 2018). Furthermore, the signal proved to be prone to extreme line broadening, when a target protein containing TMSf was titrated with a low molecular weight ligand, which may be attributed to the overall rigidity of the TMSf amino acid. To alleviate these drawbacks, we designed a tag that is identical to the TMS tag 1 (Figure 1) except that it contains an amide rather than an ester group. Following attachment to a single cysteine residue in the E. coli single-stranded DNA binding protein, the amide tag enabled the observation of intermolecular nuclear Overhauser effects (NOEs) with single-stranded DNA (Jabar et al. 2017). In other proteins, however, the tag proved prone to peak doubling, which could be attributed to cis/trans amide bond isomerization.

The new TMS tag 1 (Figure 1) solves this problem by containing a more flexible ester moiety. It can be selectively attached to solvent-exposed cysteine residues by generating a redoxstable thioether bond between tag and protein. It produces a single TMS signal at a chemical shift near 0 ppm. Its ${ }^{1} \mathrm{H}-\mathrm{NMR}$ signal is easy to detect and, owing to its chemical shift in a spectral region with few protein peaks, its intensity can readily be quantified. This makes the tag ideal for monitoring ligand binding events both in the fast and slow exchange regimes with small quantities of protein and ligand. We demonstrate the approach for two different drug targets, the Zika virus NS2B-NS3 protease (ZiPro) and the human prolyl isomerase FK506 binding protein 
(FKBP). ZiPro is closely related to the NS2B-NS3 proteases of a range of flaviviruses such as dengue and West Nile viruses, which cause regular epidemic outbreaks (Boldescu et al. 2017). The NS2B-NS3 proteases catalyze the post-translational processing of the viral precursor polyprotein into smaller protein units, which are essential for viral replication (Nitsche et al. 2014). Based on previous successes with HIV and HCV protease inhibitors, flaviviral proteases are promising drug targets, but drug development has been frustrated by the polar character of substrate-derived inhibitors, a shallow substrate binding site, and a lack of structural elucidation, and only few inhibitors have been identified for ZiPro (Boldescu et al. 2017; Nitsche et al. 2014). Human FKBP is the target of the immunosuppressive drugs tacrolimus (FK-506) and rapamycin, which suppress organ rejection after allogeneic transplantations (Siekierka et al. 1989). FKBPs are involved in multiple regulatory pathways, offering prospects for prevention and treatment of a broad range of diseases (Bonner and Boulianne 2017).

\section{Materials and Methods}

\section{Synthesis of the trimethylsilyl tag}

Details of the protocol for the chemical synthesis of the trimethylsilyl tag 1 (Figure 1) are given in the Supporting Information.

\section{Preparation of the wild type and V36C mutant of the Zika virus NS2B-NS3 protease}

The construct of the Zika virus NS2B-NS3 protease (ZiPro) has been described previously (Mahawaththa et al. 2017; Nitsche et al. 2017). Briefly, it includes 48 hydrophilic core residues of NS2B (residues $48^{*}-95^{*}$, where the star indicates residues in NS2B) followed by a $\mathrm{Gly}_{4} \mathrm{SerGly}_{4}$ linker, the N-terminal residues of the NS3 protease domain (residues 1-170), and a His6 tag. The mutations R95*A, K15N, R29G, C80S and C143S were introduced to increase stability, reduce the potential of auto-cleavage, and avoid dimerization (Lei et al. 2016; Mahawaththa et al. 2017; Nitsche et al. 2017). This construct is referred to as wild type. The mutation V36C was introduced for selective ligation of compound 1 to position 36. The expression construct used the T7 expression vector pETMCSI (Neylon et al. 2000). The plasmid was transformed into E. coli BL21(DE3) and cells were grown in LB medium containing ampicillin at $37^{\circ} \mathrm{C}$ until an $\mathrm{OD}_{600}$ value of 0.5-0.8 was reached. Subsequently, overexpression was induced with IPTG $(1 \mathrm{mM})$ and the cells were incubated overnight at room temperature. The 
cells were pelleted by centrifuging at $5000 \mathrm{~g}$ for 10 minutes and lysed by passing twice through a French Press (SLM Aminco, USA) at 830 bars. The cell lysate was centrifuged for $1 \mathrm{~h}$ at $34,000 \mathrm{~g}$ and the supernatant was loaded onto a $5 \mathrm{ml} \mathrm{Ni-NTA}$ column (GE Healthcare, USA) pre-equilibrated with loading buffer $(50 \mathrm{mM}$ Tris- $\mathrm{HCl}, \mathrm{pH} 7.5,300 \mathrm{mM} \mathrm{NaCl}, 10 \%$ glycerol). The protein was eluted with a gradually increasing concentration of elution buffer (50 mM Tris$\mathrm{HCl}$, pH 7.5, $300 \mathrm{mM} \mathrm{NaCl}, 300 \mathrm{mM}$ imidazole, 10\% glycerol), fractions were analyzed by $12 \%$ SDS-PAGE, and the buffer was exchanged to $20 \mathrm{mM}$ MES, pH $6.5,150 \mathrm{mM} \mathrm{NaCl}$.

\section{Preparation of FKBP R18C}

A construct of the human prolyl isomerase FK506 binding protein (FKBP) with C-terminal His6 tag was incorporated into a pCDF-1b vector (Novagen) using the restriction sites NcoI and NotI. The additional mutations $\mathrm{C} 22 \mathrm{~S}$ and $\mathrm{R} 18 \mathrm{C}$ were introduced for site-selective tagging of position 18 with compound $\mathbf{1}$. The plasmid was transformed into E. coli BL21(DE3) and cells were grown in LB medium containing streptomycin at $37^{\circ} \mathrm{C}$ until an $\mathrm{OD}_{600}$ value of $0.5-0.8$ was reached. Overexpression was induced with IPTG $(1 \mathrm{mM})$ and the cells were incubated overnight at room temperature. The cells were pelleted by centrifuging at $5000 \mathrm{~g}$ for 10 minutes and lysed by passing twice through a French Press at 830 bars. The cell lysate was centrifuged for $1 \mathrm{~h}$ at $34,000 \mathrm{~g}$ and the supernatant was loaded onto a $5 \mathrm{ml} \mathrm{Ni-NTA}$ column (GE Healthcare, USA) pre-equilibrated with loading buffer $(50 \mathrm{mM}$ Tris- $\mathrm{HCl}, \mathrm{pH} 7.5,300 \mathrm{mM} \mathrm{NaCl}, 10 \%$ glycerol, 5 $\mathrm{mM}$ 2-mercaptoethanol). The protein was eluted with a gradually increasing concentration of elution buffer ( $50 \mathrm{mM}$ Tris-HCl, pH 7.5, $300 \mathrm{mM} \mathrm{NaCl}, 300 \mathrm{mM}$ imidazole, $10 \%$ glycerol, 5 $\mathrm{mM}$ 2-mercaptoethanol) and fractions were analyzed by 12\% SDS-PAGE. The buffer was exchanged to buffer A (50 mM sodium phosphate buffer, $\mathrm{pH} 6.5,150 \mathrm{mM} \mathrm{NaCl}, 5 \mathrm{mM} 2-$ mercaptoethanol), and the protein was loaded onto a GE Superdex 75 (HiLoad 16/60) size exclusion column and eluted with buffer A.

\section{Tagging procedure}

ZiPro V36C and FKBP R18C samples were treated with DTT $(2 \mathrm{mM})$ for $1 \mathrm{~h}$ at room temperature, before the buffer was exchanged to buffer A (20 mM MES, pH 6.5, $150 \mathrm{mM} \mathrm{NaCl}$, $10 \%$ glycerol). $100 \mu \mathrm{M}$ samples were subsequently incubated with compound 1 (500 $\mu \mathrm{M}, 10$ 
mM DMSO stock) overnight at room temperature. The buffer was changed to NMR buffer (20 mM MES, pH 6.5, $150 \mathrm{mM} \mathrm{NaCl,} \mathrm{10 \%} \mathrm{D}_{2} \mathrm{O}$ ).

\section{NMR measurements and $K_{d}$ determination}

All protein NMR spectra were recorded in $3 \mathrm{~mm}$ NMR tubes at $25^{\circ} \mathrm{C}$, using an $800 \mathrm{MHz}$ Bruker Avance III NMR spectrometer equipped with a $5 \mathrm{~mm}$ TCI cryoprobe. Each titration step added ligand from a concentrated DMSO stock and a ${ }^{1} \mathrm{H}-\mathrm{NMR}$ spectrum was recorded with water suppression by the double spin-echo sequence (Hwang and Shaka 1995), using 512 scans per spectrum with a recovery delay of 1.3 seconds. The spectra were calibrated with respect to the water frequency. A control experiment with DMSO alone added at the concentration of the final titration point confirmed that solvent-induced chemical shift changes were insignificant. In the case of fast chemical exchange (FKBP), the dissociation constant was determined from the change in chemical shift of the TMS signal observed during titration with ligand. In the case of slow chemical exchange (ZiPro), the dissociation constant was determined from the change in relative peak intensities of the TMS signal of the complex, where the peak height of the TMS peak was divided by the overall integral of the spectral region between 0.5 and $0.9 \mathrm{ppm}$.

\section{Results and Discussion}

\section{Tag synthesis and tagging reaction}

The TMS tag 1 was readily synthesized in large quantities via only two synthetic steps (Scheme S1). The purity of the synthesized compounds was $\geq 95 \%$. Quantitative and selective alkylation of single cysteine residues in the target proteins was achieved at neutral $\mathrm{pH}$ without any additional reagents, except that the TMS tag was added dissolved in DMSO.

\section{Zika virus NS2B-NS3 protease}

To study inhibitor binding at the active site of ZiPro, we installed the TMS group close to the catalytic center formed by Ser135, His51, and Asp75 (Figure 2). Visual inspection of the crystal structure of ZiPro (PDB code: 5LC0) (Lei et al. 2016) suggested Val36 as a suitable residue for tagging, which was consequently mutated to cysteine. To avoid cross-reactivity with the solvent- 
exposed residues Cys80 and Cys143 in the wild-type amino-acid sequence, these residues were mutated to serine residues as in previous studies of ZiPro (Lei et al. 2016; Mahawaththa et al. 2017; Nitsche et al. 2017). These mutations were present in all ZiPro constructs used in this work and we refer, in the following, to the double-mutant C80S/C143S as wild type and the triple-mutant V36C/C80S/C143S as ZiPro V36C. Reduced ZiPro V36C readily reacted with the TMS tag 1 as confirmed by high-resolution mass spectrometry (Figure S2). Figure 2 illustrates the attachment site of 1 relative to the active site of ZiPro. The high solvent exposure of 1 entails high flexibility of the tag, which supports a narrow line shape of its TMS signal in the ${ }^{1} \mathrm{H}-\mathrm{NMR}$ spectrum and, therefore, high sensitivity.

To confirm that the installation of a TMS tag adjacent to the active site has no significant influence on substrate binding and enzymatic reactivity, we compared the kinetic parameters $K_{\mathrm{m}}$ and $k_{\text {cat }}$ of the tagged mutant with wild-type ZiPro (Table 1, Figure S1). Both proteins displayed similar $K_{\mathrm{m}}$ values, indicating unencumbered substrate binding. Equally, the $k_{\text {cat }}$ values were only marginally different, with a slight $k_{\text {cat }}$ increase for the tagged protease compared to the wild type, and the overall catalytic efficiencies $\left(k_{\mathrm{cat}} / K_{\mathrm{m}}\right)$ were indistinguishable. This indicates that the presence of the tag presents no significant perturbation. The ${ }^{1} \mathrm{H}-\mathrm{NMR}$ spectrum of tagged ZiPro only showed a single narrow TMS peak at about $0.06 \mathrm{ppm}$ (Figure 3) as expected for a homogeneous sample, which was subsequently used for titration with the ligand 2.

Compound 2, which is known as an active-site inhibitor of flaviviral proteases (de la Cruz et al. 2011; Nitsche et al. 2014; Brecher et al. 2017), was chosen as a model ligand to assess the performance of the TMS tag $\mathbf{1}$ in a ligand titration experiment. Inhibition experiments revealed $\mathrm{IC}_{50}$ values of about $160 \mu \mathrm{M}$ for the wild type and the tagged mutant V36C (Table 1). This value is two orders of magnitude higher than reported previously (Lim et al. 2016), but the similarities of the $\mathrm{IC}_{50}$ values determined under identical conditions for the tagged and wild type versions (Table 1) clearly indicate that the TMS tag at position 36 does not influence the intermolecular recognition and binding interaction.

Titration of tagged ZiPro V36C with compound 2 led to the appearance of a new TMS peak for the complex while the original TMS peak of the free protein weakened and disappeared (Figure 3), showing that the ligand exchanges slowly on the NMR time scale in agreement with previous observations (Mahawaththa et al. 2017). A second, smaller TMS peak appearing further up-field was attributed to a complex with the hydrolysis products of compound 2 , which is prone 
to hydrolysis in aqueous solution and proteolytic cleavage by proteases. Hydrolysis of $\mathbf{2}$ was detected by the gradual emergence of the characteristic yellow color of $p$-nitrophenolate during the titration. An additional smaller TMS peak was not observed in the titration experiments with FKBP, indicating that the ester bond of the TMS tag is stable. After $24 \mathrm{~h}$ at $25{ }^{\circ} \mathrm{C}$, however, the FKBP sample displayed two additional small TMS signals with integrals less than $10 \%$ of the main peak, which may be attributed either to partial hydrolysis of the tag or protein degradation.

Determining the $K_{\mathrm{d}}$ value in situations of slow exchange is difficult, as it requires low protein concentrations in the vicinity of the dissociation constant. This presents a problem, as the low sensitivity of NMR spectroscopy demands relatively high sample concentrations. The high sensitivity afforded by the ${ }^{1} \mathrm{H}-\mathrm{NMR}$ signal of the TMS group thus presents a clear advantage. For example, 1D NMR spectra of a $75 \mu \mathrm{M}$ solution of tagged ZiPro provided excellent signal-tonoise ratios already within a few minutes. In view of the $\mathrm{IC}_{50}$ values, we expected a dissociation constant $K_{\mathrm{d}}$ in the two- to three-digit micromolar range. In the slow exchange regime, the $K_{\mathrm{d}}$ value can be determined using

$$
I=I_{\max } \frac{\left([L]+[P]+K_{d}\right)-\sqrt{\left([L]+[P]+K_{d}\right)^{2}-4[L][P]}}{2[P]}
$$

where $I$ denotes the TMS peak intensity of the complex and $I_{\max }$ the TMS peak intensity observed for the fully saturated complex, and $[L]$ and $[P]$ are the concentrations of ligand and protein, respectively. As the intensity of only a single TMS peak is monitored, the approach is independent of its $T_{1}$ and $T_{2}$ relaxation times, so long as excess ligand does not modify the properties of the complex. Apparent peak intensities can vary, however, if the NMR sample is removed from the magnet and re-inserted for each titration step. Therefore, it proved important to reference the intensity of the TMS peak to an internal standard. While, in principle, the integral of the TMS peak of the complex could be compared with the sum of the integrals of the TMS signals of the complex and free protein, more reliable measurements were obtained by normalizing the peak height of the TMS peak of the complex (measured with local baseline correction to establish the base of the peak) by the overall integral of the methyl region of the NMR spectrum (0.5-0.9 ppm). Figure 4 shows the fit of the NMR titration data to equation (1), revealing a $K_{\mathrm{d}}$ value of $64 \pm 27 \mu \mathrm{M}$ for compound 2 . 


\section{FKBP}

To validate the utility of the tag for a different protein-ligand complex, we investigated FKBP as a second model system with two different ligands (3 and 4), which are weakly and tightly binding inhibitors. For site-specific tagging of FKBP with compound $\mathbf{1}$ adjacent to the binding site of inhibitor 3 , the natural cysteine residue in position 22 was mutated to serine and the solvent-exposed residue Arg18 was mutated to cysteine for tagging (Figure 5). FKBP R18C was readily and selectively tagged with compound $\mathbf{1}$, as confirmed by mass spectrometry (Figure S3). In contrast to the experiments conducted with ZiPro and ligand $\mathbf{2}$, ligand $\mathbf{3}$ was stable during the experiment and the TMS signal shifted gradually with increasing ligand concentration, maintaining a single peak throughout the titration experiment (Figure 6). Compound $\mathbf{3}$ has previously been identified as low-molecular weight ligand for FKBP by 2D NMR methods, where residues in the binding site highlighted in Figure 5 displayed the characteristics of fast chemical exchange (Shuker et al. 1996). Our data confirm the fast exchange situation and allow determination of the $K_{\mathrm{d}}$ value from simple 1D NMR spectra (Figure 7).

The dissociation constant of the complex of FKBP with compound $\mathbf{3}$ has been reported previously as $0.1 \mathrm{mM}$ (Shuker et al. 1996). To determine the $K_{\mathrm{d}}$ value, the titration curve of Figure 7 was fitted using

$$
\Delta \delta=\Delta \delta_{\max } \frac{\left([L]+[P]+K_{d}\right)-\sqrt{\left([L]+[P]+K_{d}\right)^{2}-4[L][P]}}{2[P]}
$$

where $\Delta \delta$ is the observed chemical shift difference, $\Delta \delta_{\max }$ the maximal chemical shift difference at saturation, and $[L]$ and $[P]$ are the concentrations of ligand and protein, respectively. The fitted curve shown in Figure 7 determined a $K_{\mathrm{d}}$ value of $94 \pm 10 \mu \mathrm{M}$, which is in excellent agreement with the value reported previously (Shuker et al. 1996).

Finally, we tested the TMS tag for its capability to monitor binding events remote from the tagging site. We chose the drug rapamycin (4), which binds to FKBP with very high affinity $\left(K_{\mathrm{i}}=0.2 \mathrm{nM}\right)$ at a site far from the TMS tag (Figure 5) (Bierer et al. 1990). Dissociation constants in the subnanomolar concentration range cannot be determined by conventional NMR experiments. The actual binding event, however, was very clearly detected by the TMS signal 
(Figure 8). The tag thus offers a sensitive probe not only for $K_{\mathrm{d}}$ determinations, but also for the detection of binding events between large biomolecules without a requirement of immediate proximity to the binding interface.

\section{Concluding remarks}

The binding affinity is a very important validation parameter for any further development of lead candidates in drug discovery. Quite generally, using NMR to monitor the target protein during titration experiments has the great advantage of reporting not only on ligand binding but also the continued integrity of the protein towards aggregation and unfolding. NMR spectroscopy of uniformly isotope-labeled proteins thus is routinely used to detect binding and measure dissociation constants, but this is difficult for very large proteins and proteins that cannot easily be enriched with stable isotopes. The chemical tag presented here capitalizes on the chemical shift of the TMS group in a spectral region that contains few ${ }^{1} \mathrm{H}-\mathrm{NMR}$ signals of the protein and therefore enables accurate measurement of peak intensities. It is easy to use, detects binding with high sensitivity, and allows the quantitative measurement of dissociation constants not only in the fast but also the slow exchange regime. In the slow exchange limit, it is necessary to quantify the peak intensity and this is difficult when the peak is not in a spectral region with minimal signal overlap, as the protein spectrum changes step-wise in response to added ligand and therefore the baseline may not always be easy to identify. We anticipate that the simplicity of the tag, tagging reaction, and NMR detection will make the TMS tag 1 popular in different stages of the drug discovery process for proteins containing a single reactive cysteine residue. Work is in progress to broaden the approach by genetic encoding of an unnatural amino acid containing a TMS group with a chemical shift near 0 ppm.

\section{Acknowledgments}

We thank Mr Mithun Chamikara Mahawaththa for mass spectrometry measurements and Professor Rolf Hilgenfeld for providing a sample of the substrate Bz-Nle-Lys-Lys-Arg-AMC. C.N. and G.O. thank the Alexander von Humboldt Foundation for a Feodor Lynen Fellowship and the Australian Research Council for a Laureate Fellowship, respectively. Financial project support by the Australian Research Council is gratefully acknowledged. Financial support by the 
Austrian Science Fund (FWF) (DK Molecular Enzymology W901 to K.Z.) and by NAWI Graz is gratefully acknowledged.

\section{References}

Arntson KE, Pomerantz WC (2016) Protein-observed fluorine NMR: A bioorthogonal approach for small molecule discovery. J Med Chem 59:5158-5171.

Bierer BE, Mattila PS, Standaert RF, Herzenberg LA, Burakoff SJ, Crabtree G, Schreiber SL (1990) Two distinct signal transmission pathways in T lymphocytes are inhibited by complexes formed between an immunophilin and either FK506 or rapamycin. Proc Natl Acad Sci USA 87:9231-9235.

Boldescu V, Behnam MAM, Vasilakis N, Klein CD (2017) Broad-spectrum agents for flaviviral infections: dengue, Zika and beyond. Nat Rev Drug Discov 16:565-586.

Bonner JM, Boulianne GL (2017) Diverse structures, functions and uses of FK506 binding proteins. Cell Signal 38:97-105.

Brecher M, Li Z, Liu B, Zhang J, Koetzner CA, Alifarag A, Jones SA, Lin Q, Kramer LD, Li H (2017) A conformational switch high-throughput screening assay and allosteric inhibition of the flavivirus NS2B-NS3 protease. PLOS Pathog 13:e1006411.

Cellitti SE, Jones DH, Lagpacan L, Hao X, Zhang Q, Hu H, Brittain SM, Brinker A, Caldwell J, Bursulaya B, Spraggon G, Brock A, Ryu Y, Uno T, Schultz PG, Geierstanger BH (2008) In vivo incorporation of unnatural amino acids to probe structure, dynamics, and ligand binding in a large protein by nuclear magnetic resonance spectroscopy. J Am Chem Soc 130:9268-9281.

Chen WN, Kuppan KV, Lee MD, Jaudzems K, Huber T, Otting G (2015) O-tert-Butyltyrosine, an NMR tag for high-molecular-weight systems and measurements of submicromolar ligand binding affinities. J Am Chem Soc 137:4581-4586.

Chen WN, Nitsche C, Pilla KB, Graham B, Huber T, Klein CD, Otting G (2016) Sensitive NMR approach for determining the binding mode of tightly binding ligand molecules to protein targets. J Am Chem Soc 138:4539-4546.

de la Cruz L, Nguyen TH, Ozawa K, Shin J, Graham B, Huber T, Otting G (2011) Binding of low molecular weight inhibitors promotes large conformational changes in the dengue virus NS2B-NS3 protease: fold analysis by pseudocontact shifts. J Am Chem Soc 133:19205-19215.

Doak BC, Norton RS, Scanlon MJ (2016) The ways and means of fragment-based drug design. Pharmacol Ther 167:28-37.

Gossert AD, Jahnke W (2016) NMR in drug discovery: A practical guide to identification and validation of ligands interacting with biological macromolecules. Prog Nucl Magn Reson Spectrosc 97:82-125. 
Hwang TL, Shaka AJ (1995) Water suppression that works. Excitation sculpting using arbitrary wave-forms and pulsed-field gradients. J Magn Reson A 112:275-279.

Jabar S, Adams LA, Wang Y, Aurelio L, Graham B, Otting G (2017) Chemical tagging with tert-butyl and trimethylsilyl groups for measuring intermolecular nuclear Overhauser effects in a large protein-ligand complex. Chem Eur J 23:13033-13036.

Jackson JC, Hammill JT, Mehl RA (2007) Site-specific incorporation of a ${ }^{19} \mathrm{~F}$-amino acid into proteins as an NMR probe for characterizing protein structure and reactivity. $\mathrm{J}$ Am Chem Soc 129:1160-1166.

Lei J, Hansen G, Nitsche C, Klein CD, Zhang L, Hilgenfeld R (2016) Crystal structure of Zika virus NS2B-NS3 protease in complex with a boronate inhibitor. Science 353:503-505.

Lim L, Roy A, Song J (2016) Identification of a Zika NS2B-NS3pro pocket susceptible to allosteric inhibition by small molecules including qucertin rich in edible plants. bioRxiv.

Liu JJ, Horst R, Katritch V, Stevens RC, Wüthrich K (2012) Biased signaling pathways in $\beta_{2}$ adrenergic receptor characterized by ${ }^{19} \mathrm{~F}-\mathrm{NMR}$. Science 335:1106-1110.

Loh C-T, Adams LA, Graham B, Otting G (2018) Genetically encoded amino acids with tertbutyl and trimethylsilyl groups for site-selective studies of proteins by NMR spectroscopy. J Biomol NMR:doi: 10.1007/s10858-10017-10157-y.

Mahawaththa MC, Pearce BJG, Szabo M, Graham B, Klein CD, Nitsche C, Otting G (2017) Solution conformations of a linked construct of the Zika virus NS2B-NS3 protease. Antiviral Res 142:141-147.

Neylon C, Brown SE, Kralicek AV, Miles CS, Love CA, Dixon NE (2000) Interaction of the Escherichia coli replication terminator protein (Tus) with DNA: A model derived from DNA-binding studies of mutant proteins by surface plasmon resonance. Biochemistry 39:11989-11999.

Nitsche C, Holloway S, Schirmeister T, Klein CD (2014) Biochemistry and medicinal chemistry of the dengue virus protease. Chem Rev 114:11348-11381.

Nitsche C, Mahawaththa MC, Becker W, Huber T, Otting G (2017) Site-selective tagging of proteins by pnictogen-mediated self-assembly. Chem Commun 53:10894-10897.

Nitsche C, Otting G (2018) NMR studies of ligand binding. Curr Opin Struct Biol 48:16-22.

Shuker SB, Hajduk PJ, Meadows RP, Fesik SW (1996) Discovering high-affinity ligands for proteins: SAR by NMR. Science 274:1531-1534.

Siekierka JJ, Hung SH, Poe M, Lin CS, Sigal NH (1989) A cytosolic binding protein for the immunosuppressant FK506 has peptidyl-prolyl isomerase activity but is distinct from cyclophilin. Nature 341:755-757. 
Table 1. Kinetic and inhibition parameters of wild-type ZiPro and ZiPro V36C tagged with the TMS probe 1.

\begin{tabular}{|c|c|c|c|c|}
\hline & $K_{\mathbf{m}}$ & $k_{\text {cat }}$ & $\boldsymbol{k}_{\text {cat }} / \boldsymbol{K}_{\mathrm{m}}$ & $\mathrm{IC}_{50}$ \\
\hline & $(\mu \mathbf{M})$ & $\left(\mathrm{s}^{-1}\right)$ & $\left(10^{6} \cdot s^{-1} \cdot M^{-1}\right)$ & $(\mu \mathbf{M})^{\mathrm{d}}$ \\
\hline $\operatorname{ZiPro}(w t)^{\mathrm{a}}$ & $2.4 \pm 0.3$ & $8.6 \pm 0.1$ & $3.6(3.2-4.1)^{\mathrm{c}}$ & $160 \pm 8$ \\
\hline $\operatorname{ZiPro}(V 36 C)+\operatorname{tag} 1^{b}$ & $2.5 \pm 0.3$ & $10.5 \pm 0.4$ & $4.2(3.7-4.9)^{\mathrm{c}}$ & $158 \pm 22$ \\
\hline
\end{tabular}

${ }^{a}$ Zika virus protease $\mathrm{C} 80 \mathrm{~S} / \mathrm{C} 143 \mathrm{~S}$

${ }^{\mathrm{b}}$ Zika virus protease C80S/C143S/V36C with covalently attached tag 1 at position 36.

${ }^{c}$ Uncertainty range determined by error propagation.

${ }^{\mathrm{d}}$ Half maximal inhibitory concentration of compound 2 at $10 \mu \mathrm{M}$ substrate concentration. 


\section{Figures}

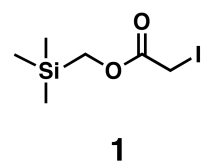<smiles>N=C(N)Nc1ccc(C(=O)Oc2ccc([N+](=O)[O-])cc2)cc1</smiles>

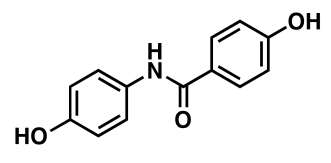

3

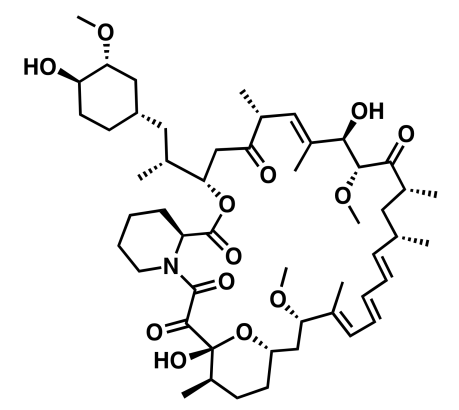

4

Figure 1. Chemical structures of the TMS tag and ligands used in this study. 1: Cysteinereactive TMS tag. 2: 4-Nitrophenyl 4-guanidinobenzoate, which is an active-site inhibitor of the Zika virus NS2B-NS3 protease (ZiPro). 3: 4-Hydroxy- $N$-(4-hydroxyphenyl)benzamide, which is a low-affinity FKBP ligand. 4: Rapamycin, which is a high-affinity FKBP ligand.

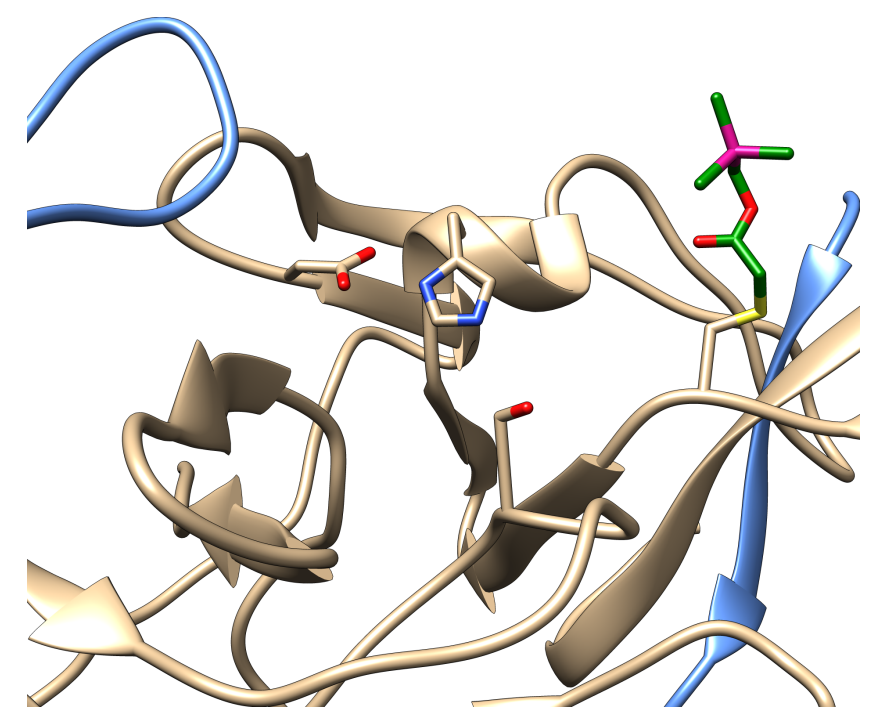

Figure 2. Active site of the Zika virus NS2B-NS3 protease showing the TMS tag 1 (green) attached at position 36 and the side-chains of the catalytic triad (Ser135, His51, Asp75). Sulfur is highlighted in yellow, oxygen in red, nitrogen in blue, and silicon in magenta. NS3 is shown in beige and NS2B in blue. 


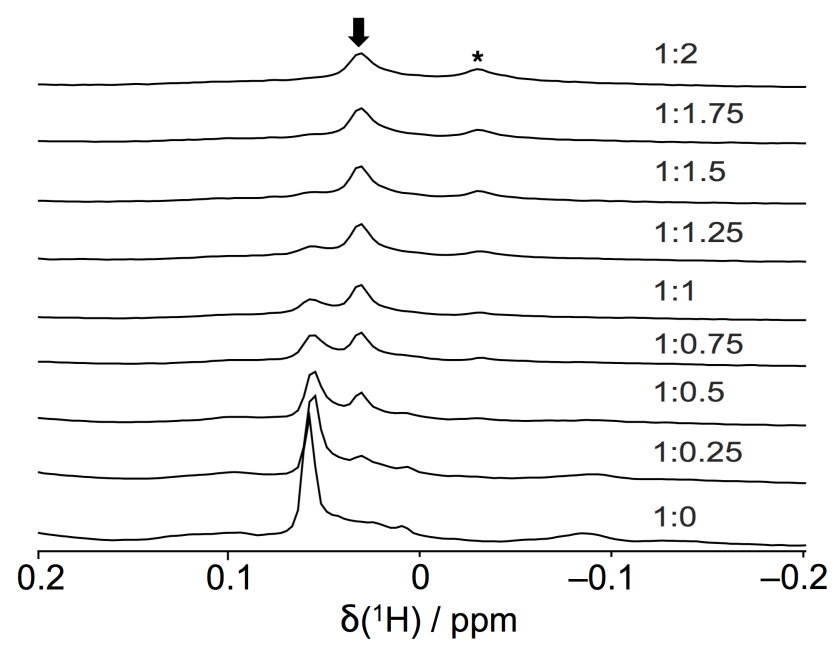

Figure 3. 1D ${ }^{1} \mathrm{H}-\mathrm{NMR}$ spectra monitoring the titration of ZiPro $(75 \mu \mathrm{M}$ in $20 \mathrm{mM}$ MES buffer, $\mathrm{pH}$ 6.5, containing $150 \mathrm{mM} \mathrm{NaCl}$ ) with inhibitor 2. The spectra were recorded on an $800 \mathrm{MHz}$ NMR spectrometer. We assign the original TMS peak at $0.06 \mathrm{ppm}$ and the new peak at $0.03 \mathrm{ppm}$ (labeled with an arrow and used to calculate the binding affinity, see Figure 4) to free ZiPro and ZiPro in complex with $\mathbf{2}$, respectively. The star identifies a minor species attributed to an interaction with hydrolyzed compound $\mathbf{2}$. The titration ratios of protein to ligand are indicated. 


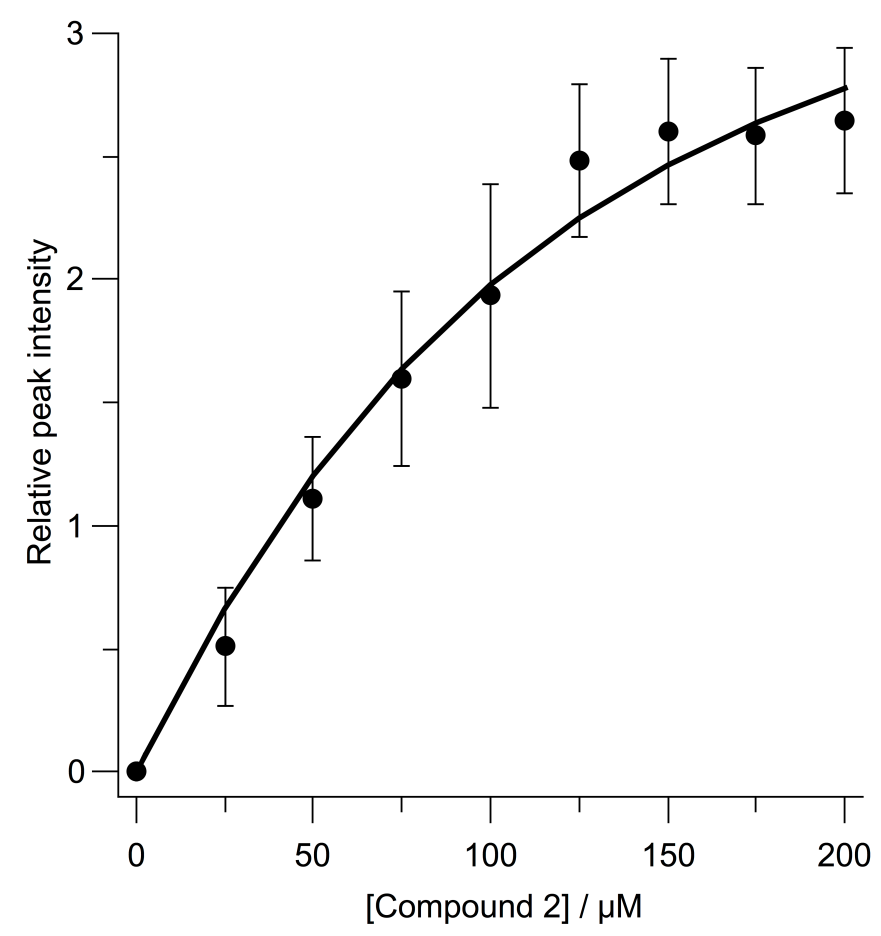

Figure 4. Titration curve for ZiPro and inhibitor $\mathbf{2}$ based on the NMR spectra shown in Figure 3. The peak intensity of the new TMS peak (identified by an arrow in Figure 3) against the concentration of compound 2 . The fit of equation (1) determined a $K_{\mathrm{d}}$ value of $64 \pm 27 \mu \mathrm{M}$. Errors were calculated with respect to signal-to-noise ratios.

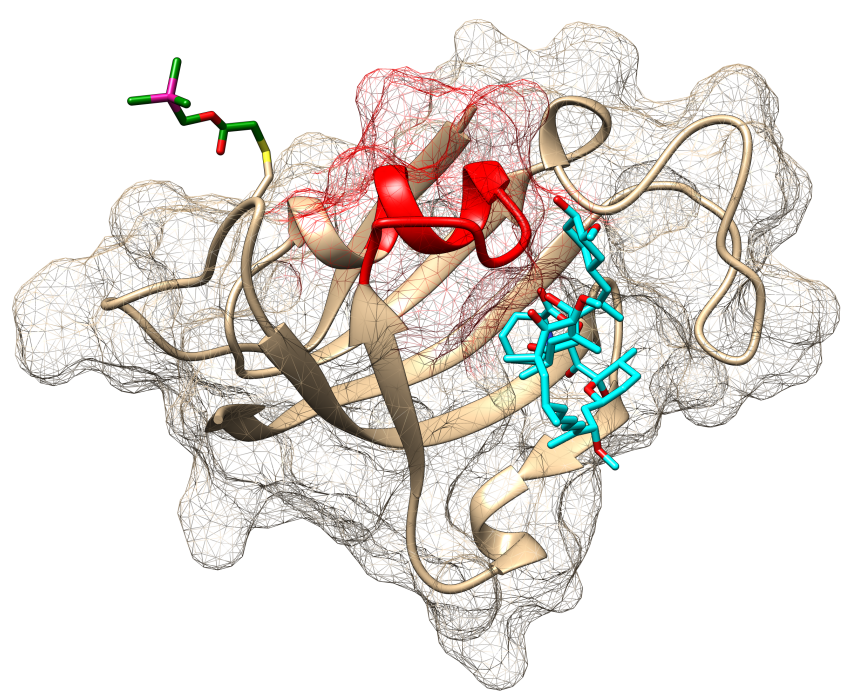

Figure 5. Structure of FKBP modeled with the TMS tag $\mathbf{1}$ (green) attached to R18C. Sulfur is highlighted in yellow, oxygen in red, nitrogen in blue, and silicon in magenta. The binding site for inhibitor $\mathbf{3}$ is highlighted in red. Rapamycin (4) is shown in cyan. 


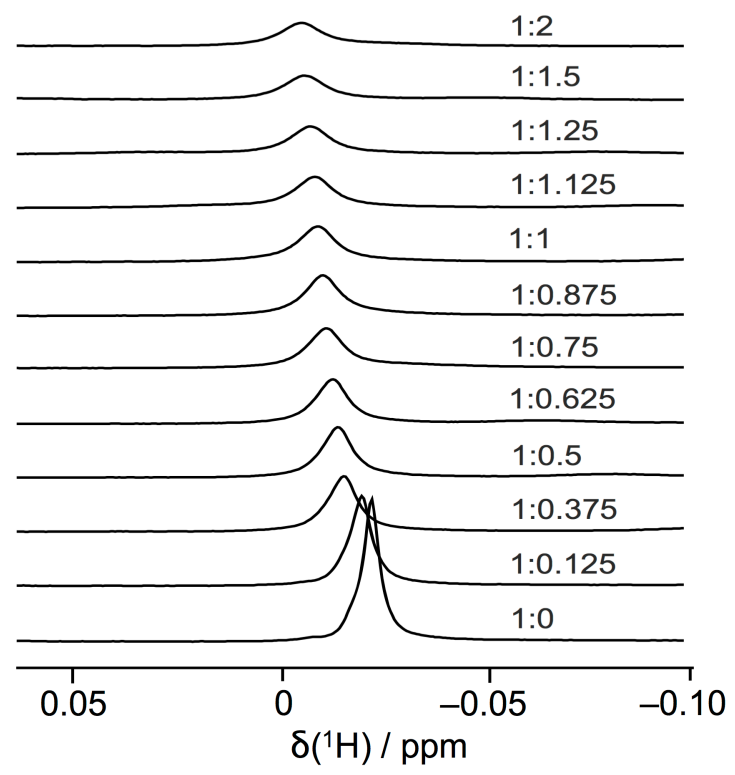

Figure 6. 1D ${ }^{1} \mathrm{H}-\mathrm{NMR}$ spectra of a $130 \mu \mathrm{M}$ solution of FKBP in $20 \mathrm{mM}$ MES buffer, pH 6.5, and $150 \mathrm{mM} \mathrm{NaCl}$, monitoring the titration with ligand 3. The spectra were recorded on an 800 MHz NMR spectrometer. The gradual change in the chemical shift of the TMS peak was used to calculate the binding affinity (Figure 7). The titration ratios of protein to ligand are indicated. 


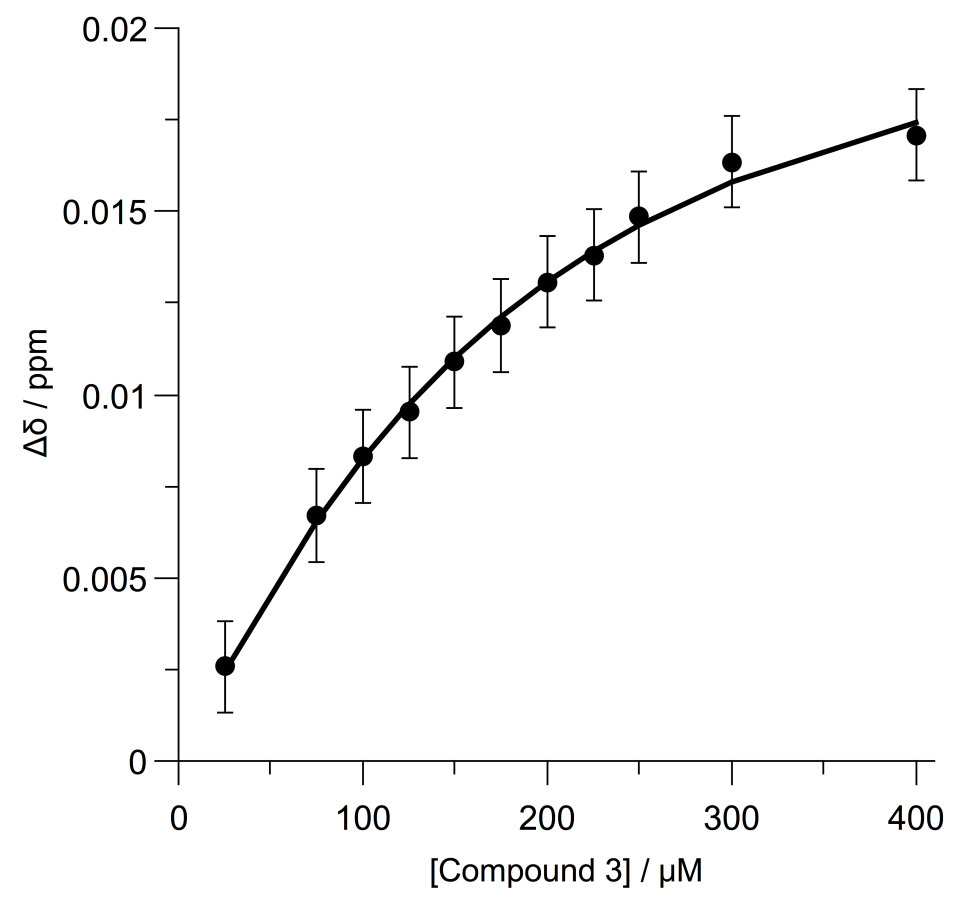

Figure 7. Titration curve plotting the chemical shift changes observed for the TMS peak in response to increasing concentrations of compound 3 (Figure 6). A fit of equation (2) determined a $K_{\mathrm{d}}$ value of $94 \pm 10 \mu \mathrm{M}$. Error bars reflect an estimated uncertainty in chemical shift of $\pm 1 \mathrm{~Hz}$.

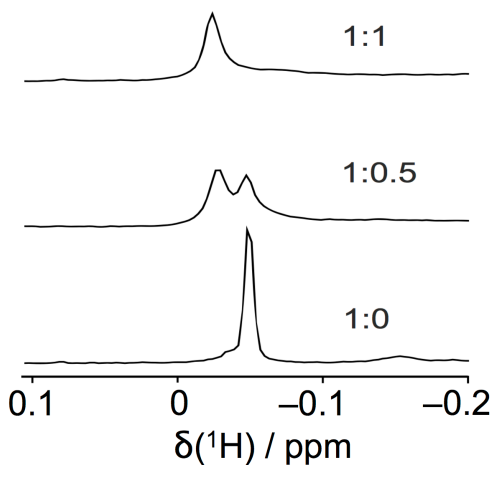

Figure 8. 1D ${ }^{1} \mathrm{H}-\mathrm{NMR}$ spectra of FKBP with different ratios of FKBP to the tightly binding ligand rapamycin (4), using the same sample conditions and NMR spectrometer as in Figure 6. 